\title{
Autophagy is activated in the ovarian tissue of polycystic ovary syndrome
}

\author{
Da $\mathrm{Li}^{1}$, Yue You ${ }^{2}$, Fang-Fang Bi ${ }^{2}$, Tie-Ning Zhang ${ }^{3}$, Jiao Jiao ${ }^{1}$, Tian-Ren Wang ${ }^{1,4}$, Yi-Ming Zhou ${ }^{5}$, \\ Zi-Qi Shen ${ }^{1}$, Xiu-Xia Wang ${ }^{1}$ and Qing Yang ${ }^{2}$ \\ ${ }^{1}$ Center of Reproductive Medicine, Shengjing Hospital of China Medical University, Shenyang, China, \\ ${ }^{2}$ Department of Obstetrics and Gynecology, Shengjing Hospital of China Medical University, Shenyang, China, \\ ${ }^{3}$ Department of Pediatrics, Shengjing Hospital of China Medical University, Shenyang, China, ${ }^{4}$ Department of \\ Obstetrics, Gynecology, and Reproductive Sciences, Yale School of Medicine, New Haven, Connecticut, USA \\ and ${ }^{5}$ Department of Medicine, Brigham and Women's Hospital, Harvard Institutes of Medicine, Harvard Medical \\ School, Boston, Massachusetts, USA
}

Correspondence should be addressed to Q Yang or X-X Wang; Email: yangq@sj-hospital.org or wangxxsj@sina.cn

\begin{abstract}
The importance of autophagy in polycystic ovary syndrome (PCOS)-related metabolic disorders is increasingly being recognized, but few studies have investigated the role of autophagy in PCOS. Here, transmission electron microscopy demonstrated that autophagy was enhanced in the ovarian tissue from both humans and rats with PCOS. Consistent with this, ovarian granulosa cells from PCOS rats showed increases in the autophagy marker protein light chain 3B (LC3B), whereas levels of the autophagy substrate SQSTM1/p62 were decreased. In addition, the ratio of LC3-II/LC3-I was markedly elevated in human PCOS ovarian tissue compared with normal ovarian tissue. Real-time PCR arrays indicated that 7 and 34 autophagy-related genes were down- and up-regulated in human PCOS , Signal-Net, and regression analysis suggested that there are a wide range of interactions among these 41 genes, and a potential network based on EGFR, ERBB2, FOXO1, MAPK1, NFKB1, IGF1, TP53 and MAPK9 may be responsible for autophagy activation in PCOS. Systematic functional analysis of 41 differential autophagy-related genes indicated that these genes are highly involved in specific cellular processes such as response to stress and stimulus, and are linked to four significant pathways, including the insulin, ERBB, mTOR signaling pathways and protein processing in the endoplasmic reticulum. This study provides evidence for a potential role of autophagy disorders in PCOS in which autophagy may be an important molecular event in the pathogenesis of PCOS.

Reproduction (2018) 155 85-92
\end{abstract}

\section{Introduction}

Polycystic ovary syndrome (PCOS) is a common endocrine disorder affecting approximately $10 \%$ of women of reproductive age (Goodarzi et al. 2011), with significant and diverse reproductive, metabolic (obesity, insulin resistance, type 2 diabetes, adverse cardiovascular risk profiles) and psychological features (Teede et al. 2010, Chan et al. 2017). In addition to a genetic predisposition, environmental and lifestyle factors contribute to the pathogenesis of PCOS (Insenser et al. 2013, Chan et al. 2017).

Autophagy is a major degradation pathway responsive to energy stress and is necessary for cellular homeostasis to maintain energy production and synthesis of new macromolecules (Delbridge et al. 2017, Jacob et al. 2017). Notably, the importance of autophagy in PCOSrelated metabolic disorders is increasingly being recognized. For example, autophagy has an important role in the control of inflammasome activation in response to metabolic stress (Lee et al. 2016). In obesity, autophagy genes are upregulated in human adipose tissue and autophagy is activated and associated with adipose dysfunction (Maixner et al. 2016). Furthermore, macrophage autophagy has a crucial role in the regulation of systemic insulin sensitivity in obesity (Kang et al. 2016). Several autophagic markers are inhibited in skeletal muscle in severely insulin-resistant patients with type 2 diabetes, and long-term high doses of insulin may generate a vicious cycle of insulin resistance through inhibiting autophagy (Moller et al. 2017). In addition, dysregulation of autophagy is frequently associated with cardiomyopathy and cardiac dysfunction during obesity and diabetes (Wang et al. 2017).

However, to date, the precise biological effects underlying mechanisms of autophagy in PCOS development remain largely unknown. To further elucidate the relationship between autophagy and PCOS, the present study investigated the autophagy and autophagy-related molecular network in human PCOS ovarian tissue and a PCOS rat model. 


\section{Materials and methods}

\section{Ethical statement}

The study was conducted in accordance with ethical standards and the Helsinki Declaration of 1975.

\section{Patients and tissue collection}

This study was approved by the Institutional Review Board at China Medical University, and informed written consent was obtained from all patients prior to the initiation of the study. From October 2014 to September 2016, normal ovarian tissue $(n=10)$ was obtained during the follicular phase from women with regular menstrual cycles who were younger than 40 years of age and underwent operative laparoscopy or laparotomy (as part of investigation and/or treatment of infertility or other gynecological disease). PCOS ovarian tissue $(n=10)$ was obtained during the follicular phase from women with irregular menstrual cycles who were younger than 40 years of age and underwent laparoscopic wedge resection. PCOS was diagnosed according to the 2003 Rotterdam criteria (Rotterdam 2004).

\section{Animal model and sample collection}

All experiments were conducted according to the NIH Guide for Care and Use of Laboratory Animals and all experimental procedures involving animals were approved by the Animal Care and Use Committee of China Medical University. Female Sprague Dawley rats (3 weeks old) were purchased from the Beijing HFK Bioscience Co. Ltd. The rats were housed under standard laboratory conditions $\left(22 \pm 2{ }^{\circ} \mathrm{C}, 12 / 12 \mathrm{~h}\right.$ light/ darkness cycle with lights on from $6: 00 \mathrm{~h}$ to $18: 00 \mathrm{~h}$ ) and given ad libitum access to food and water. After one week of acclimatization, the rats were randomly divided into two groups: a control group and a dehydroepiandrosterone (DHEA) group (each group, $n=6$ ). The PCOS rat model was established by injection of DHEA dissolved in $0.2 \mathrm{~mL}$ sesame oil $(60 \mathrm{mg} / \mathrm{kg}$ body weight, sc) with a high-fat diet (nutrients comprised $45 \%$ kcal fat, 35\% kcal carbohydrate and $20 \%$ kcal protein), and the control group was subcutaneously injected with an equivalent amount of sesame oil (Zhang et al. 2016). Body weight was recorded weekly and fasting blood was collected from the abdominal aorta under anesthesia at the end of week 12. Histological samples of the ovary were placed in 4\% paraformaldehyde for haematoxylin-eosin (H\&E) staining and immunohistochemistry, and 2.5\% glutaraldehyde for transmission electron microscopy (TEM), respectively.

\section{Real-time PCR array}

Total RNA was extracted using Trizol reagent (Invitrogen) according to the manufacturer's protocol. DNA contamination was removed by adding DNase I (Invitrogen). For the quantification of 224 key genes involved in autophagy, total RNA was then reverse-transcribed from $2 \mu \mathrm{g}$ of RNA using the PrimeScript RT Reagent Kit with gDNA Eraser (TaKaRa) and amplified by GoTaq qPCR Master Mix (Promega) in the ABI ViiA 7 Real-time PCR system (Applied Biosystems). The specific primer sequences are listed in Supplementary Table 1 (see section on supplementary data given at the end of this article). GAPDH mRNA was amplified as an internal control for the normalization of each sample. All samples were analyzed using the $2^{-\Delta \Delta C T}$ method.

\section{Immunohistochemistry for LC3B and SQSTM1/p62}

The autophagy markers protein light chain 3B (LC3B) and autophagy substrate SQSTM1/p62 in the ovarian tissue of the control and PCOS rats were determined by immunohistochemical staining. Briefly, serial $4-\mu \mathrm{m}$ sections were obtained from each paraffin-embedded tissue block. Following deparaffinization and rehydration, sections were subjected to microwave antigen retrieval. The primary antibodies were an anti-LC3B antibody (ab48394) (1: 200; Abcam) and an anti-SQSTM1/p62 antibody (ab91526) (1: 200; Abcam), and the sections were incubated overnight at $4{ }^{\circ} \mathrm{C}$ with this antibody. 3,3'-diaminobenzidine was used as the chromogen. Nuclei were counterstained with haematoxylin, and slides were dried and mounted. Negative controls were incubated with PBS instead of the antibody. The ovarian tissue was treated with rapamycin $(500 \mathrm{nM})$ for $6 \mathrm{~h}$, which served as a positive control. The immunostaining was evaluated by two independent pathologists, who were blinded to the identity of the subject groups. Area quantification was performed with a light microscope at a magnification of 400 $\times$ and analyzed using Image-Pro Plus 6.0 (Media Cybernetics, Rockville, MD, USA). The immunoreactivity of LC3B and SQSTM1/p62 was evaluated according to the intensity and percentage of positively stained cells. The percentage of positively stained cells was graded as follows: negative, 0-29\%; weak, 30-59\%; strong, >60\%. Any borderline values were classified into the higher category.

\section{Western blotting for LC3-II/LC3-I and beclin-1}

The autophagy markers LC3-II/LC3-I and essential autophagy gene beclin-1 in human normal and PCOS ovarian tissues were measured by western blotting. Briefly, $50 \mu \mathrm{g}$ protein was separated on $12 \%$ SDS polyacrylamide gels and transferred to polyvinyl difluoride membranes (Millipore). The membranes were blocked in TBS containing $0.1 \%$ Tween-20 and 5\% non-fat dry milk for $60 \mathrm{~min}$ at room temperature and incubated with antibodies against LC3A/B (\#12741) (1:1000; Cell Signaling Technology) and beclin-1 (ab207612) (1:2000; Abcam) overnight at $4^{\circ} \mathrm{C}$. Next, the membranes were washed in PBS-Tween followed by $1 \mathrm{~h}$ incubation at room temperature with horseradish peroxidaseconjugated secondary antibody (1:5000; Santa Cruz Biotechnology) and detected using enhanced chemiluminescence.

\section{TEM}

Ovarian tissue was cut into $1 \mathrm{~mm}^{3}$ pieces and then treated with $2.5 \%$ glutaraldehyde in PBS overnight at $4{ }^{\circ} \mathrm{C}$, followed by postfixation in $1 \%$ buffered $\mathrm{OsO}_{4}$ for $3 \mathrm{~h}$, washed thrice in PBS and then dehydrated with a series of ethanol concentrations $(50,70$ and $90 \%$ ) at 15-min intervals. The samples were subsequently incubated in a mixture of alcohol and acetone ( $v: v$ 1:1) for $20 \mathrm{~min}$, followed by incubation with pure acetone for $20 \mathrm{~min}$ at $4{ }^{\circ} \mathrm{C}$. Ovarian tissue sections were then embedded in resin and polymerized. Ultrathin $(70 \mathrm{~nm})$ sections were post-stained 
with uranyl acetate and lead citrate, and examined under a Jeol 1230 electron microscope (Jeol, Akishima, Tokyo, Japan).

\section{Molecular network analysis}

STRING analysis (https://www.string-db.org) was used to identify direct and indirect interactions. STRING is a database that provides associations from computational prediction and other databases for interaction analysis. The STRING score was calculated from the combination of all predictions and network analysis was set at a medium confidence (0.400).

\section{Data visualization and statistical analysis}

The STRING database was used to identify the functional enrichments in the network. Gene Ontology (GO) and Kyoto Encyclopedia of Genes and Genomes (KEGG) annotations were also used. R packages pheatmap and ggplot2 were used for data visualization. Kolmogorov-Smirnov was used to test normality. A Bonferroni correction was used to minimize the false positive rate. Regression analysis was used to examine the possible relationship between the differentially expressed genes. The data are presented as means \pm S.D. Statistical differences in the data were evaluated by Student's $t$-test or one-way ANOVA as appropriate, and were considered significant at $P<0.05$.

\section{Results}

Active autophagy was observed in the ovarian tissue of PCOS rats

LC3 and LC3-II/LC3-I are reliable markers of autophagy. Autophagosomes can be observed using a transmission electron microscope and are considered the gold standards of autophagy activation. The rat model of PCOS exhibited polycystic ovaries (Fig. 1A). Ultrastructural observation using TEM demonstrated that autophagy was induced in the ovarian cortex of PCOS rats. In contrast, autophagosomes were rarely observed and autolysosomes were never observed in control rats (Fig. 1B). Consistent with this, ovarian granulosa cells of PCOS rats showed an increase in the autophagy marker LC3B (Fig. 1Ci and Cii), whereas levels of autophagy substrate SQSTM1/p62 were decreased (Fig. 1Di and Dii).

\section{Active autophagy was observed in human PCOS ovarian tissue}

TEM demonstrated that autophagy was enhanced in the ovarian tissue of patients with PCOS (Fig. 2A). Similarly, the ratio of LC3-II/LC3-I was markedly elevated in the PCOS group compared with the normal group (Fig. 2B), and there was a slightly increasing trend in expression of the essential autophagy protein beclin-1 (Fig. 2B).

\section{A potent autophagy-related gene network was activated in human PCOS ovarian tissue}

To further confirm the potential molecular basis of autophagy in PCOS, the expressions of 224 key genes involved in autophagy were detected by a real-time PCR array applied to the human ovarian cortex of normal and PCOS samples. The results indicated that 41 genes were differentially expressed between normal and PCOS groups (Fig. 3A and B). STRING analysis indicated a wide range of interactions among these 41 genes (Fig. 3C and Supplementary Table 2). Interestingly, both STRING and Signal-Net analyses indicated that a potential network based on EGFR, ERBB2, FOXO1, MAPK1, NFKB1, IGF1, TP53 and MAPK9 may be responsible for autophagy activation in PCOS (Fig. 3C and Supplementary Fig. 1). In addition, our data showed a highly significant correlation $(R>0.95, P<0.000001)$ between ATG16L and SIRT2/NBR1 levels (Fig. 3D and E), and HDAC1 and NBR1/MBTPS2 levels (Fig. 3F and D). A significant correlation was also observed between the expressions of another 36 pairs of genes $(R>0.90, P<0.000001)$ (Supplementary Table 3). Therefore, these results suggest that a potent autophagy-related gene network is widely activated in human PCOS ovarian tissue.

\section{Systematic functional analysis of 41 active autophagy- related genes in human PCOS ovarian tissue}

To define the functional significance of the extensive changes in autophagy-related genes in PCOS, GO analysis was performed, which revealed distinct functional categories for the PCOS-associated autophagy gene candidates. As shown in Fig. 4Ai and Supplementary Table 4, 41 differentially expressed genes were found to be highly involved in specific cellular processes, such as response to stress, external stimulus, endogenous stimulus and extracellular stimulus. STRING analysis indicated that 29 autophagyrelated proteins were widely involved in response to stress, and identified EGFR, TP53, IGF1, ERBB2 and C12orf44 as the most important nodes in the stress response network (interaction node $>5$ ) (Fig. 4Aii and Supplementary Table 5). Notably, protein binding, macromolecular complex binding, enzyme binding and purine ribonucleotide binding were the major molecular functions of these differentially expressed genes (Fig. 4Bi and Supplementary Table 6), and ERBB2, IGF1, NFKB1, TP53, ATIC, MAPK1 and EGFR were the key regulators for protein binding (interaction node $>5$ ) (Fig. 4Bii and Supplementary Table 7). In addition, TP53, NFKB1 and MAPK 1 have a crucial role in the membrane structure of the organelles in the cytosol (Fig. $4 \mathrm{Ci}$ and Cii, Supplementary Tables 8 and 9). To investigate key pathways linked to these distinct autophagy-related genes, the interaction of the significant pathways associated with PCOS was built according to the KEGG database (Fig. 4D and Supplementary Table 10). Our analysis showed that these differentially expressed genes are linked to four significant pathways, including the insulin, ERBB, mTOR signaling pathways and protein processing in the endoplasmic reticulum.

Reproduction (2018) 155 85-92 

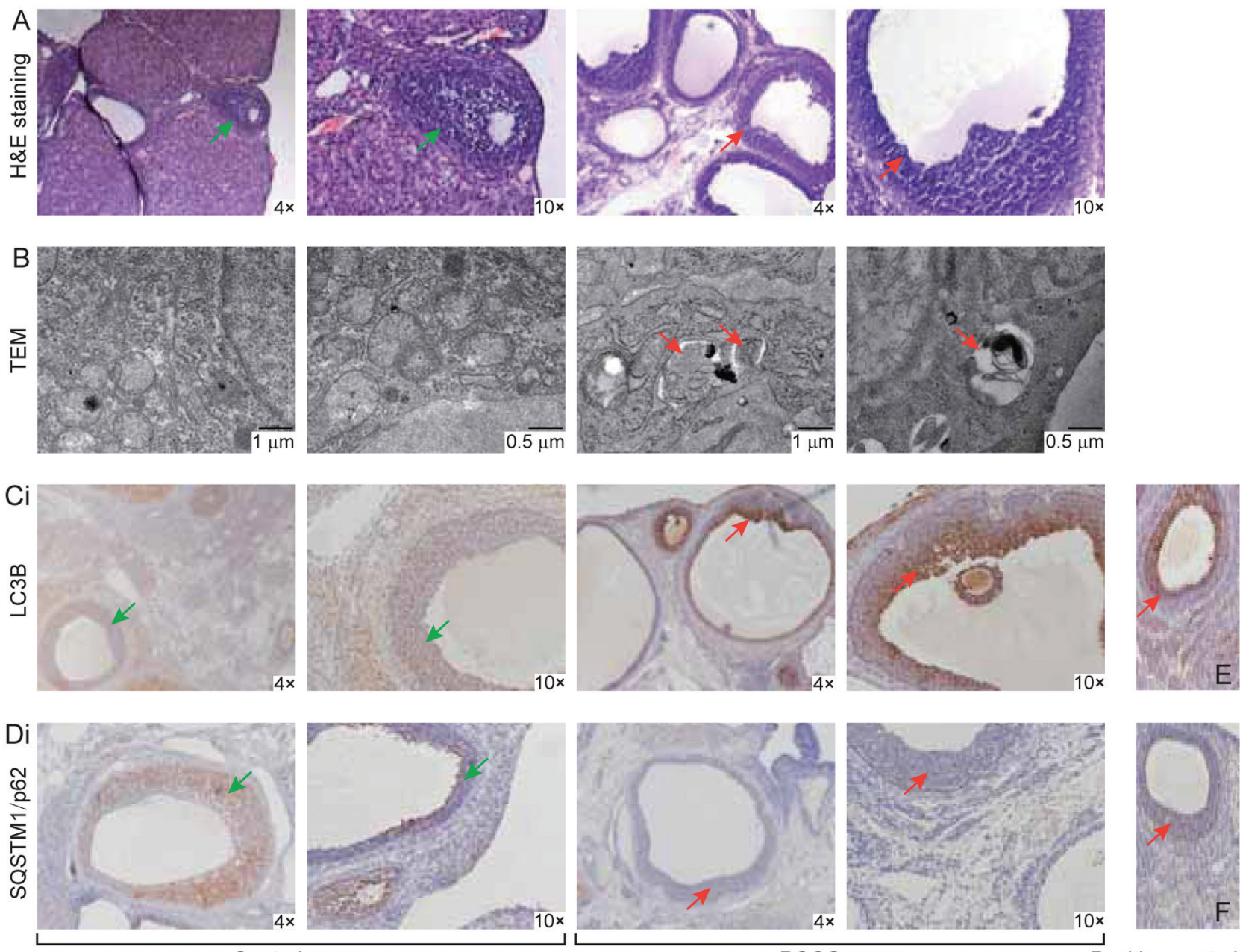

Control group

PCOS group

Positive control
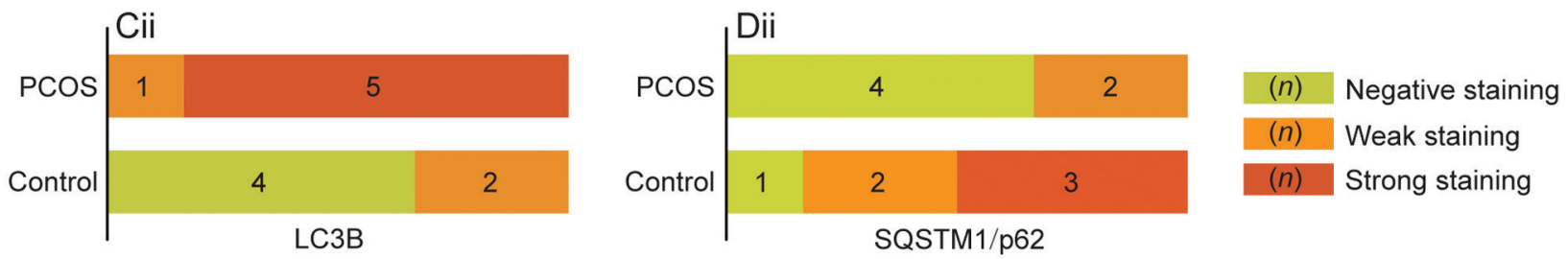

Figure 1 Ovarian morphology and autophagy levels in the ovarian tissue of control rats and PCOS rats. (A) Representative sections of H\&E staining from the ovarian tissue of rats in the control group and PCOS group, respectively. Magnification is 40x and 100x. (B) The ultrastructure was analyzed using transmission electron microscopy. Asterisks point to autophagosomes $(1 \mu \mathrm{m})$ and autolysosomes $(0.5 \mu \mathrm{m})$. (Ci and $\mathrm{Di})$ Sections were subjected to immunostaining for LC3B and SQSTM1/p62, respectively. Each group had $n=6$. (Cii and Dii) Summary of the percentage of positively stained cells from the measurements shown in $\mathrm{Ci}$ and $\mathrm{Di}$, respectively. (E and F) Positive control. H\&E, haematoxylineosin; TEM, transmission electron microscopy.

\section{Discussion}

In this study, we reported for the first time that autophagy was activated in the ovarian tissue of humans and rats with PCOS. A potential autophagy-related gene network based on EGFR, ERBB2, FOXO1, MAPK1, NFKB1, IGF1, TP53 and MAPK9 may be responsible for autophagy activation in PCOS. To date, although there is no direct evidence for a role of autophagy in PCOS, some insight was gained by a previous study showing that (i) EGFR was differentially expressed and is a potential cause of PCOS oocytes and cumulus cell disorder at early stages (Liu et al. 2016); (ii) variants in/ near ERBB2/HER2 are associated with PCOS at or near genome-wide significance (Day et al. 2015); (iii) FOXO1 was significantly increased in PCOS cumulus cells (Shi et al. 2015) and phospho-ERK1/2 were decreased in PCOS granulosa cells (Lan et al. 2015), and alteration of ERK1/2 signaling is important for follicle assembly, 


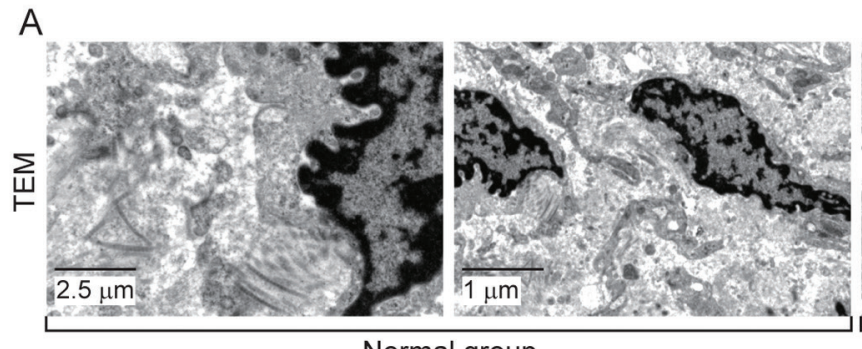

Normal group

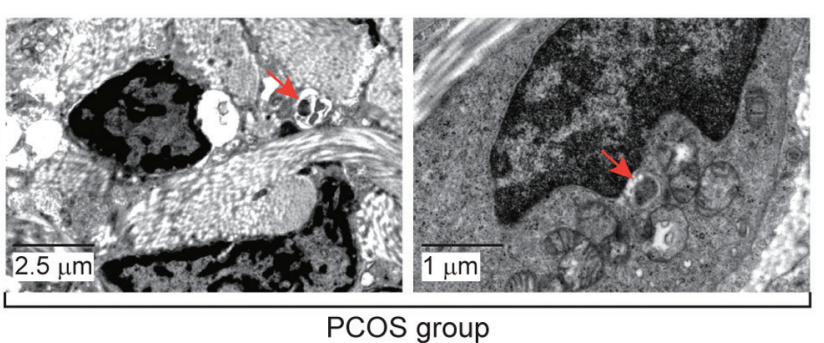

PCOS group
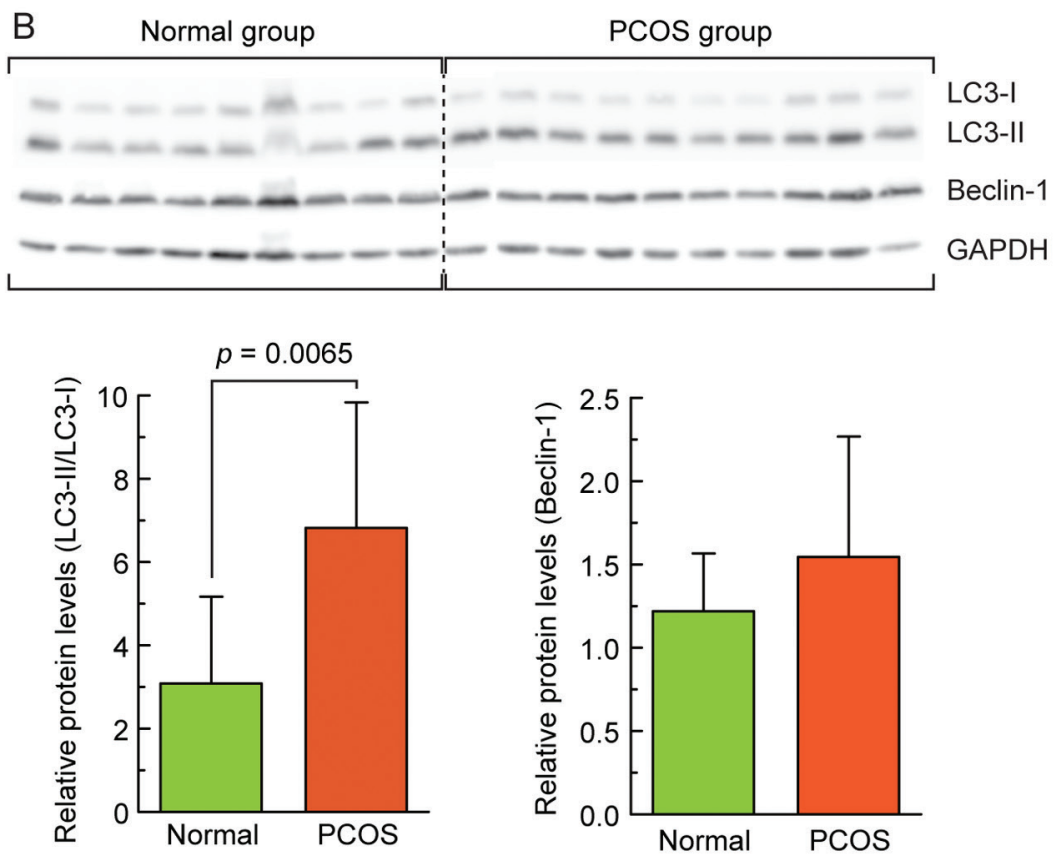

Figure 2 Ovarian autophagy levels in human normal and PCOS ovarian tissues. (A) Ultrastructure was analyzed using transmission electron microscopy. Asterisks point to autolysosomes. (B) Relative protein levels of beclin-1 and LC3-II/LC3-I were detected by western blotting ( $n=9$ for the normal group, and $n=10$ for the PCOS group). Bar graphs show mean \pm S.D. TEM, transmission electron microscopy.

further changing the rate of assembly of primordial follicles (Nilsson et al. 2013); (iv) Serum levels of NFKB1 in the PCOS groups were significantly higher than in the control group (Liu et al. 2015); (v) IGF1 is an important intermediate for follicular development and the pathogenesis of PCOS (Roche et al. 2016), cumulus cells from women with PCOS were characterized by abnormal expression of EGFR and IGF-like family proteins (IGF1R, IGF2R, IGF2BP2 and IGFBP2) (Haouzi et al. 2012) and a supraphysiological IGF1 concentration can decrease TP53 protein expression in the trophectoderm (Velazquez et al. 2011). However, to the best of our knowledge, there is still no report regarding MAPK9 in PCOS.

Notably, these observations further correlate autophagy-related molecules and processes with physiological and pathological features of PCOS. For example, activation of EGFR on granulosa cells is a key event involved in ovulation (El-Hayek et al. 2014).
$\mathrm{PI3K/AKT/FOXO1/mTOR} \mathrm{signaling} \mathrm{plays} \mathrm{a} \mathrm{critical} \mathrm{role}$ in regulating the interaction among androgens, insulin, insulin-like growth factors and hyperglycemic diet in acne (Ju et al. 2017). Furthermore, conditional ablation of the IGF1 receptor leads to increased adiposity and insulin resistance (Riera et al. 2017).

There is also preliminary evidence showing changes in the expression of autophagy genes in endometria from women with PCOS (Sumarac-Dumanovic et al. 2017). This evidence, when combined with our observation of ovarian autophagy activation in human and rats with PCOS, suggests that multiple organ autophagy changes may be a possible factor involved in the development of PCOS and PCOS-related metabolic disorders.

This study provides preliminary evidence for a potential role of autophagy disorders in PCOS, and understanding that the autophagy mechanisms involved in PCOS may yield new insight into the pathophysiology of the disorder. 
A

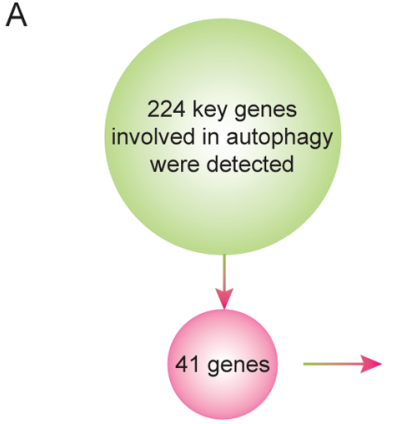

were differentially expressed between human normal and PCOS ovarian tissue
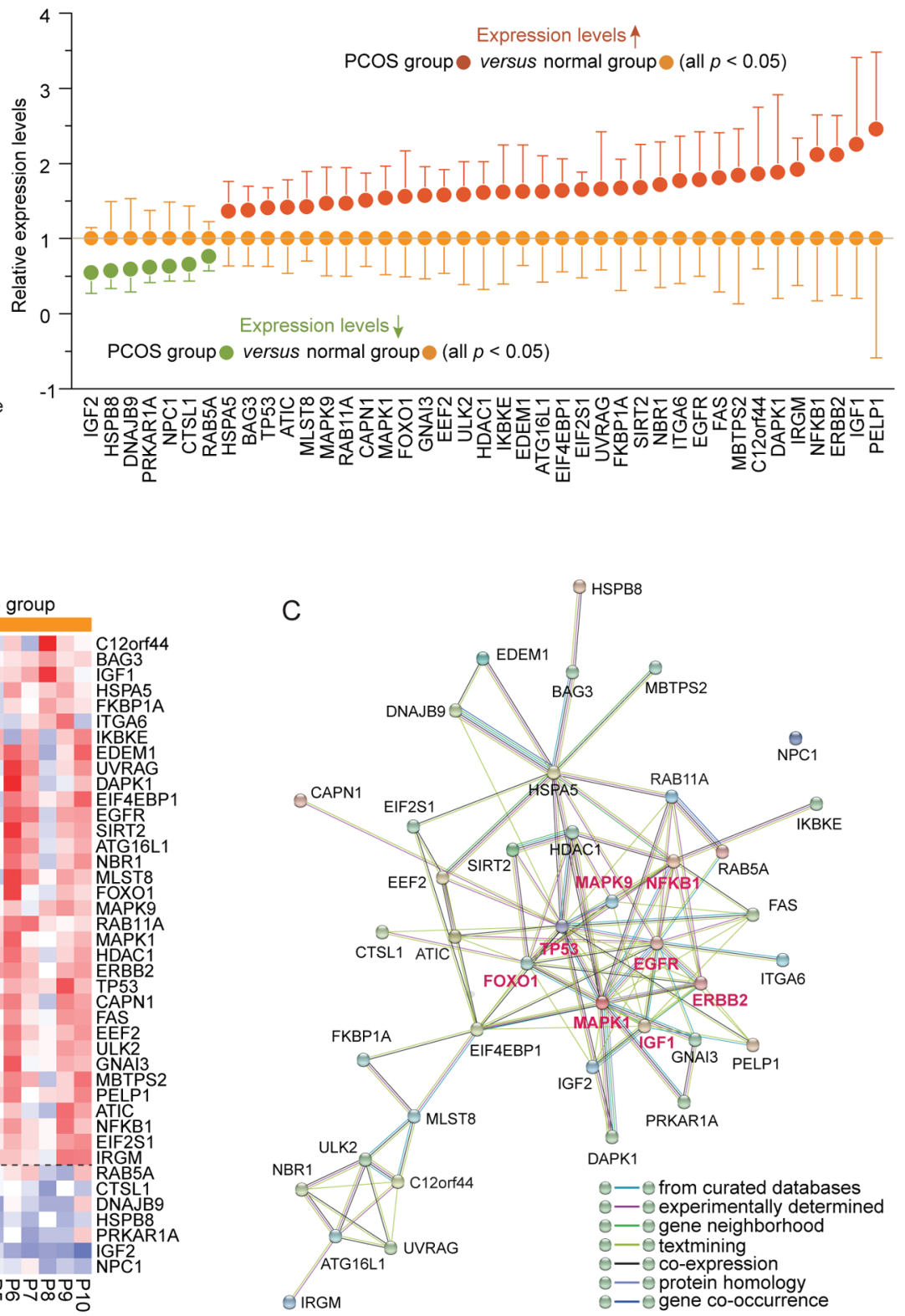

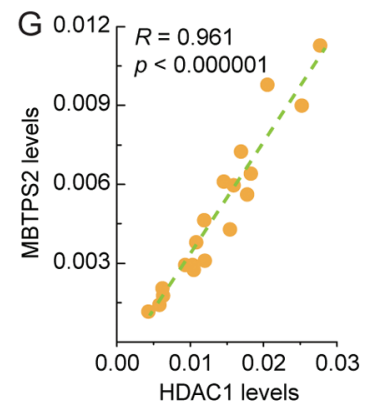

Figure 3 Intracellular levels and interactions of autophagy-related genes in human normal and PCOS ovarian tissues. (A and B) Differences in expression patterns of 41 autophagy-related genes in the human ovarian cortex of the normal and PCOS samples (each group, $n=10)$. (C) STRING analysis displayed the interactions of these 41 autophagy-related genes. (D, E, F and G) Correlation between ATG16L and SIRT2 levels/ NBR1 levels, or HDAC1 and NBR1 levels/MBTPS2 levels, respectively (each group, $n=20$ ). Bar graphs show mean \pm S.D. 
$\mathrm{Ai}$

Biological Process (GO)
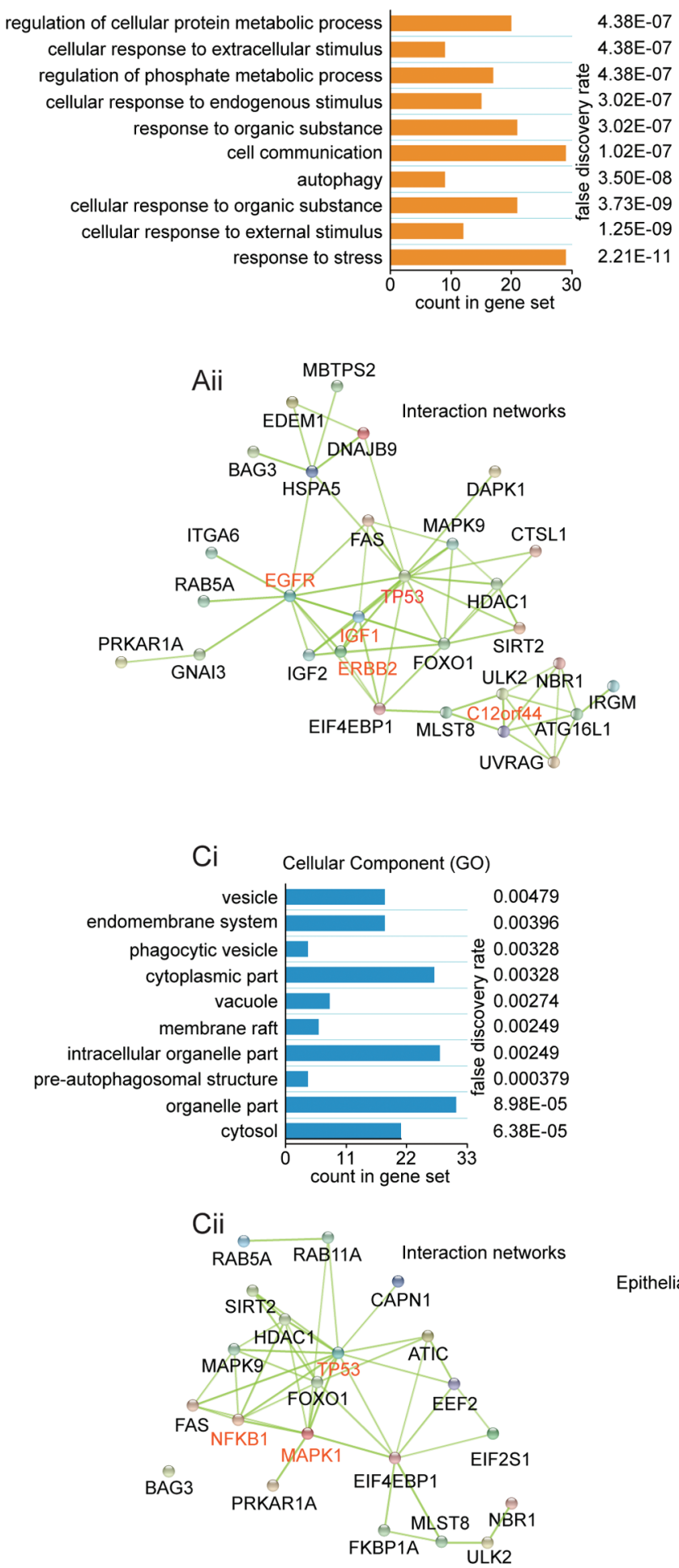

$\mathrm{Bi} \quad$ Molecular Function (GO)
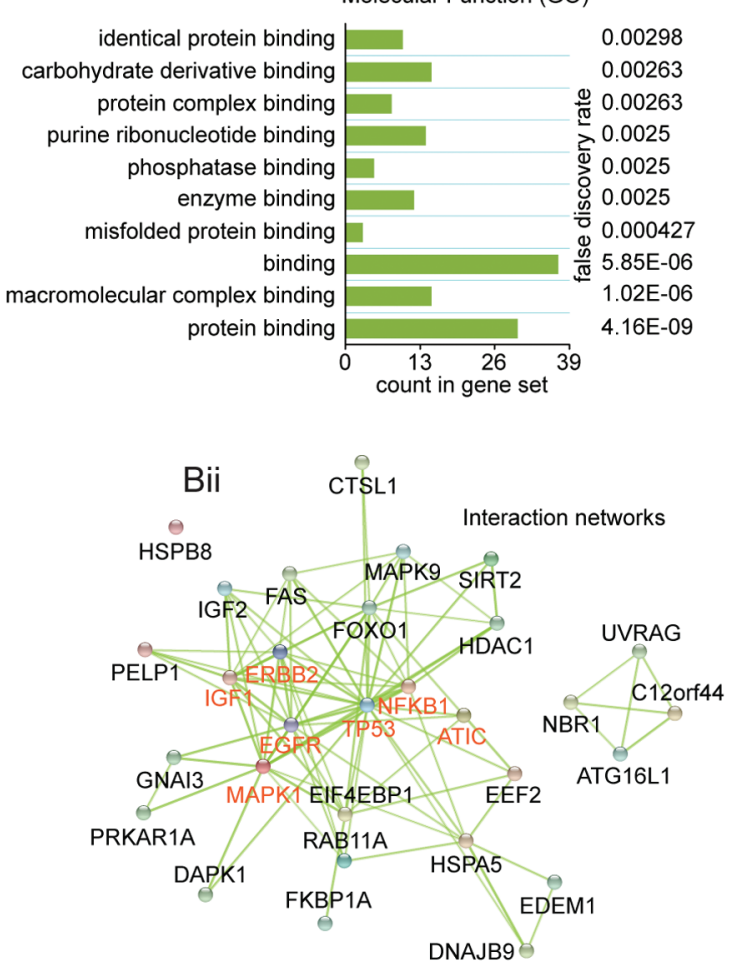

D

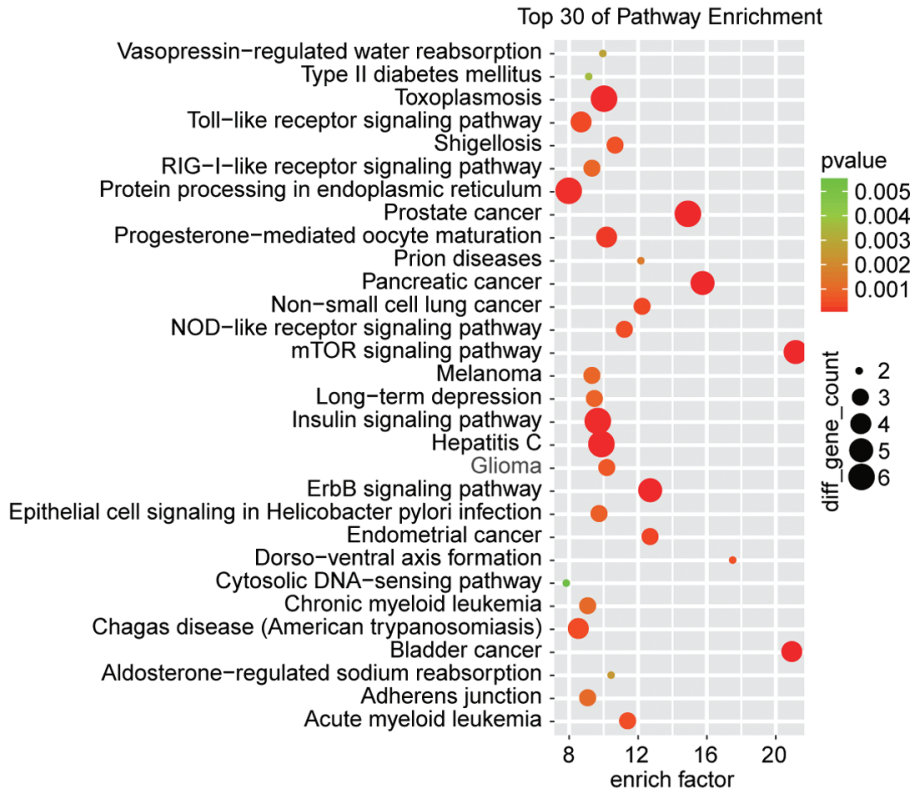

Figure 4 GO, STRING and KEGG analyses of 41 differentially expressed autophagy-related genes in human PCOS ovarian tissue. (Ai, Aii, Bi, Bii and $\mathrm{Ci}$ ) Biological Process, Molecular Function and Cellular Component analysis of 41 differentially expressed autophagy-related genes between human normal and PCOS ovarian tissues, respectively. The top $10 \mathrm{GO}$ terms are shown. (Aii, Bi, Bii, Ci and Cii) STRING analysis displaying the interactions of the top functional enrichments in Biological Process, Molecular Function and Cellular Component, respectively. (D) KEGG analysis of 41 differentially expressed autophagy-related genes between human normal and PCOS ovarian tissues, respectively. The top 30 KEGG terms are shown. 


\section{Supplementary data}

This is linked to the online version of the paper at https://doi.org/10.1530/REP-17-0499.

\section{Declaration of interest}

The authors declare that there is no conflict of interest that could be perceived as prejudicing the impartiality of the research reported.

\section{Funding}

This work was supported by the National Natural Science Foundation of China (81671423, 81402130 and 81501247), Clinical Capability Construction Project for Liaoning Provincial Hospitals (LNCCC-A01-2015), Fok Ying Tung Education Foundation (151039), Campus Research Fund of China Medical University (YQ20160004) and Distinguished Talent Program of Shengjing Hospital (No. ME76).

\section{Acknowledgements}

The authors would like to thank Yuan-Yuan Fang for her insight and assistance in the preparation of this manuscript.

\section{References}

Chan JL, Kar S, Vanky E, Morin-Papunen L, Piltonen T, Puurunen J, Tapanainen JS, Rosa Maciel GA, Hayashida SAY, Soares JM et al. 2017 Racial and ethnic differences in the prevalence of metabolic syndrome and its components of metabolic syndrome in women with polycystic ovary syndrome: a regional cross-sectional study. American Journal of Obstetrics and Gynecology 217 189.e1-189.e8. (https://doi. org/10.1016/j.ajog.2017.04.007)

Day FR, Hinds DA, Tung JY, Stolk L, Styrkarsdottir U, Saxena R, Bjonnes A, Broer L, Dunger DB, Halldorsson BV et al. 2015 Causal mechanisms and balancing selection inferred from genetic associations with polycystic ovary syndrome. Nature Communications 6 8464. (https:// doi.org/10.1038/ncomms9464)

Delbridge LMD, Mellor KM, Taylor DJ \& Gottlieb RA 2017 Myocardial stress and autophagy: mechanisms and potential therapies. Nature Reviews Cardiology 14 412-425. (https://doi.org/10.1038/nrcardio.2017.35)

El-Hayek S, Demeestere I \& Clarke HJ 2014 Follicle-stimulating hormone regulates expression and activity of epidermal growth factor receptor in the murine ovarian follicle. PNAS 111 16778-16783. (https://doi. org/10.1073/pnas.1414648111)

Goodarzi MO, Dumesic DA, Chazenbalk G \& Azziz R 2011 Polycystic ovary syndrome: etiology, pathogenesis and diagnosis. Nature Reviews Endocrinology 7 219-231. (https://doi.org/10.1038/nrendo.2010.217)

Haouzi D, Assou S, Monzo C, Vincens C, Dechaud H \& Hamamah S 2012 Altered gene expression profile in cumulus cells of mature MII oocytes from patients with polycystic ovary syndrome. Human Reproduction $\mathbf{2 7}$ 3523-3530. (https://doi.org/10.1093/humrep/des325)

Insenser M, Montes-Nieto R, Murri M \& Escobar-Morreale HF 2013 Proteomic and metabolomic approaches to the study of polycystic ovary syndrome. Molecular and Cellular Endocrinology 370 65-77. (https:// doi.org/10.1016/j.mce.2013.02.009)

Jacob JA, Salmani JMM, Jiang Z, Feng L, Song J, Jia X \& Chen B 2017 Autophagy: An overview and its roles in cancer and obesity. Clinica Chimica Acta 468 85-89. (https://doi.org/10.1016/j.cca.2017.01.028)

Ju Q, Tao T, Hu T, Karadag AS, Al-Khuzaei S \& Chen W 2017 Sex hormones and acne. Clinics in Dermatology 35 130-137. (https://doi.org/10.1016/j. clindermatol.2016.10.004)

Kang YH, Cho MH, Kim JY, Kwon MS, Peak JJ, Kang SW, Yoon SY \& Song Y 2016 Impaired macrophage autophagy induces systemic insulin resistance in obesity. Oncotarget 7 35577-35591. (https://doi. org/10.18632/oncotarget.9590)

Lan CW, Chen MJ, Tai KY, Yu DC, Yang YC, Jan PS, Yang YS, Chen HF \& Ho HN 2015 Functional microarray analysis of differentially expressed genes in granulosa cells from women with polycystic ovary syndrome related to MAPK/ERK signaling. Scientific Reports 5 14994. (https://doi. org/10.1038/srep14994)

Lee HY, Kim J, Quan W, Lee JC, Kim MS, Kim SH, Bae JW, Hur KY \& Lee MS 2016 Autophagy deficiency in myeloid cells increases susceptibility to obesity-induced diabetes and experimental colitis. Autophagy 12 1390-1403. (https://doi.org/10.1080/15548627.2016.1184799)

Liu M, Gao J, Zhang Y, Li P, Wang H, Ren X \& Li C 2015 Serum levels of TSP-1, NF-kappaB and TGF-beta1 in polycystic ovarian syndrome (PCOS) patients in northern China suggest PCOS is associated with chronic inflammation. Clinical Endocrinology 83 913-922. (https://doi. org/10.1111/cen.12951)

Liu Q, Li Y, Feng Y, Liu C, Ma J, Li Y, Xiang H, Ji Y, Cao Y, Tong X \& Xue Z 2016 Single-cell analysis of differences in transcriptomic profiles of oocytes and cumulus cells at GV, MI, MII stages from PCOS patients. Scientific Reports 6 39638. (https://doi.org/10.1038/srep39638)

Maixner N, Bechor S, Vershinin Z, Pecht T, Goldstein N, Haim Y \& Rudich A 2016 Transcriptional dysregulation of adipose tissue autophagy in obesity. Physiology 31 270-282. (https://doi.org/10.1152/ physiol.00048.2015)

Moller AB, Kampmann U, Hedegaard J, Thorsen K, Nordentoft I, Vendelbo MH, Moller N \& Jessen N 2017 Altered gene expression and repressed markers of autophagy in skeletal muscle of insulin resistant patients with type 2 diabetes. Scientific Reports 7 43775. (https://doi. org/10.1038/srep43775)

Nilsson E, Zhang B \& Skinner MK 2013 Gene bionetworks that regulate ovarian primordial follicle assembly. BMC Genomics 14 496. (https:// doi.org/10.1186/1471-2164-14-496)

Riera CE, Tsaousidou E, Halloran J, Follett P, Hahn O, Pereira MMA, Ruud LE, Alber J, Tharp K, Anderson CM et al. 2017 The sense of smell impacts metabolic health and obesity. Cell Metabolism 26 198-211. (https://doi.org/10.1016/j.cmet.2017.06.015)

Roche J, Rame C, Reverchon M, Mellouk N, Cornuau M, Guerif F, Froment P \& Dupont J 2016 Apelin (APLN) and Apelin Receptor (APLNR) in human ovary: expression, signaling, and regulation of steroidogenesis in primary human luteinized granulosa cells. Biology of Reproduction 95 104. (https://doi.org/10.1095/biolreprod.116.141754)

Rotterdam EA-SPCWG 2004 Revised 2003 consensus on diagnostic criteria and long-term health risks related to polycystic ovary syndrome. Fertility and Sterility 81 19-25. (https://doi.org/10.1016/j.fertnstert.2003.10.004)

Shi L, Liu S, Zhao W \& Shi J 2015 miR-483-5p and miR-486-5p are down-regulated in cumulus cells of metaphase II oocytes from women with polycystic ovary syndrome. Reproductive BioMedicine Online 31 565-572. (https://doi.org/10.1016/j.rbmo.2015.06.023)

Sumarac-Dumanovic $M$, Apostolovic $M$, Janjetovic $K$, Jeremic $D$, Popadic D, Ljubic A, Micic J, Dukanac-Stamenkovic J, Tubic A, Stevanovic D et al. 2017 Downregulation of autophagy gene expression in endometria from women with polycystic ovary syndrome. Molecular and Cellular Endocrinology 440 116-124. (https://doi.org/10.1016/j. mce.2016.11.009)

Teede H, Deeks A \& Moran L 2010 Polycystic ovary syndrome: a complex condition with psychological, reproductive and metabolic manifestations that impacts on health across the lifespan. BMC Medicine 8 41. (https:// doi.org/10.1186/1741-7015-8-41)

Velazquez MA, Hermann D, Kues WA \& Niemann H 2011 Increased apoptosis in bovine blastocysts exposed to high levels of IGF1 is not associated with downregulation of the IGF1 receptor. Reproduction 141 91-103. (https://doi.org/10.1530/REP-10-0336)

Wang F, Jia J \& Rodrigues B 2017 Autophagy, metabolic disease, and pathogenesis of heart dysfunction. Canadian Journal of Cardiology 33 850-859. (https://doi.org/10.1016/j.cjca.2017.01.002)

Zhang H, Yi M, Zhang Y, Jin H, Zhang W, Yang J, Yan L, Li R, Zhao Y \& Qiao J 2016 High-fat diets exaggerate endocrine and metabolic phenotypes in a rat model of DHEA-induced PCOS. Reproduction 151 431-441. (https://doi.org/10.1530/REP-15-0542)

Received 8 August 2017

First decision 8 September 2017

Revised manuscript received 4 October 2017

Accepted 13 October 2017 